\title{
Negotiating Consent: Exploring Ethical Issues when Therapeutic Massage Bodywork Practitioners Are Trained in Multiple Therapies
}

\author{
Antony J. Porcino, $\mathrm{PhD},{ }^{1 *}$ Stacey A. Page, $\mathrm{PhD},{ }^{2}$ Heather S. Boon, $\mathrm{PhD},{ }^{3}$ Marja J. Verhoef, $\mathrm{PhD}^{4}$ \\ ${ }^{1}$ School of Nursing, Faculty of Applied Sciences, University of British Columbia, Vancouver, BC, Canada, \\ ${ }^{2}$ Office of Medical Bioethics, Faculty of Medicine, University of Calgary, Calgary, AB, Canada, \\ ${ }^{3}$ Leslie Dan Faculty of Pharmacy, University of Toronto, Toronto, ON, Canada, \\ ${ }^{4}$ Department of Community Health Sciences, Faculty of Medicine, University of Calgary, Calgary, AB, Canada
}

\begin{abstract}
Introduction: Obtaining informed consent from competent patients is essential to the ethical delivery of health care, including therapeutic massage and bodywork (TMB). The informed consent process used by TMB practitioners has not been previously studied. Little information is available about the practice of informed consent in a treatment-focused environment that may involve multiple decision points, use of multiple TMB therapies, or both.

Methods: As part of a larger study on the process of providing TMB therapy, 19 practitioners were asked about obtaining informed consent during practice. Qualitative description was used to analyze discussions of the consent process generally, and about its application when practitioners use multiple TMB therapies.
\end{abstract}

Results: Two main consent approaches emerged, one based on a general consent early in the treatment process, and a second ongoing consent process undertaken throughout the course of treatment. Both processes are constrained by how engaged a patient wants to be, and the amount of information and time needed to develop a truly informed consent.

Conclusions: An understanding-based consent process that accommodates an acknowledged information differential between the patient and practitioner, and that is guided by clearly delineated goals within a trust-based relationship, may be the most effective consent process under the conditions of real practice conditions.

KEY WORDS: complementary therapies/methods; massage; musculoskeletal manipulations; consent; decision-making; qualitative research; clinical practice

\section{INTRODUCTION}

In the health services context, informed consent consists of the voluntary agreement of a competent individual to receive treatment based on an adequate understanding of the treatment's nature, purpose, and implications ${ }^{(1-5)}$. The process and practice of informed consent is grounded in the ethical principle of respect for autonomy and requires health service practitioners to meet three basic conditions. These are: (a) that the patient be fully informed as to the proposed treatment including its purpose, risks and benefits; (b) that the patient is capable of understanding the information provided; and (c) that the decision to undergo treatment is being made free from undue influence ${ }^{(2,6)}$. These conditions result in numerous legal and moral obligations for health service practitioners which have been explored from many perspectives within the field of health services ${ }^{(2,4,5)}$. Helping patients who wish to receive therapeutic massage and bodywork (TMB) to provide informed consent is problematic because the risks are usually based on hypothetical risks, or known risks specific to compromised or vulnerable populations ${ }^{(7)}$, and the benefits of many TMB are not well established. Explanation of the benefits is often based on a practitioner's or professional body's experience. Additionally, therapy sessions may incorporate multiple TMB therapies. TMB discussions are usually focused on TMB as a physical service, but informed consent in TMB must also address the potential mental and emotional risks of harm from TMB.

Informed consent for TMB treatment involves ensuring that patients understand the results of the practitioner's assessment and the subsequent therapeutic possibilities, processes, and the risks and benefits of each choice, as they would for any other health care service $^{(8)}$. Such consent either involves explicit and detailed statements, or actions by the patient that imply consent during treatment, including surrender of their decision-making autonomy to the practitioner ${ }^{(8)}$.

Obtaining informed consent for TMB services is among the competency standards for most North American TMB practitioner certification bodies and associations - for example, those of the $\mathrm{Na}$ tional Certification Board of Therapeutic Massage and Bodywork ${ }^{(9)}$, the Massage Therapy Body of Knowledge collaborative ${ }^{(10)}$, and the College of Massage Therapy of British Columbia ${ }^{(11)}$. Most TMB 
associations additionally reference the requirement of obtaining informed consent in their codes of ethics or codes of conduct - for example, those of the American Massage Therapy Association ${ }^{(12)}$ and the Massage Therapist Association of Alberta ${ }^{(13)}$. Several books and courses on ethics specific to TMB are also available ${ }^{(14-18)}$. These TMB resources on ethics highlight that consent should be informed, and provide guidance about what types of information and decision-support are relevant for informed consent. The discussions of risks in these sources include nonphysical aspects such as mental or emotional safety. However, the consent requirements within the reviewed TMB standards of practice or codes of ethics stop short of providing specific details on obtaining or maintaining informed consent in clinical practice that may involve multiple therapies, in-the-moment decisions, or needed changes to the treatment plans based on what is found during the course of treatment ${ }^{(19)}$. The language additionally can be construed to only require a single or highly constrained instance of consent, as often occurs in standard medical practice, such as for a prescription or surgery. Thus, while the importance of informed consent, both knowledge and practice, is addressed, there is limited information for practitioners regarding the practical implementation of or effectiveness for achieving consent in TMB practice, and little research on the effectiveness of the standards or the ability of TMB practitioners to operationalize informed consent during the course of practice. The studies by Caspi et al. ${ }^{(20,21)}$ confirm that this is a problem.

Most practitioners are trained in multiple TMB therapies ${ }^{(22)}$. Informed consent could be applied to each form of therapy provided by a TMB practitioner. However, there is little information indicating if, or how well, TMB practitioners are taught to obtain informed consent or if they do so when they provide multiple TMB therapies in a single session. This raises several questions about what is relevant to patients, while ensuring that patient autonomy is being respected: (a) How and when is consent obtained? (b) Should patients give consent at every decision point or change of TMB therapy during treatment, and how would those decisions points or therapy changes be recognized? (c) Are patient preferences solicited regarding their degree of decision-making involvement? (d) Do patients understand enough about the therapies and treatments they will be receiving? (e) Do patients feel free to ask questions?

As part of a research project involving interviews with TMB practitioners on the process of practice ${ }^{(22)}$, the topic of consent for treatment arose during the first interview when the practitioner described her intake process. This prompted interest in exploring negotiating consent when applying multiple therapies in a single treatment session. After the topic arose in the second interview, a specific question was added to the interview guide to further explore the issue of negotiating informed consent during the provision of multiple TMB therapies by a single therapist.

While this article focuses on the provision of TMB therapies (the term used here to describe any of the myriad forms of massage used for therapeutic effect during a therapeutic encounter), many of the practitioners provide non-TMB therapies as well, including various forms of energy work, shamanism or counseling, and devices (e.g., laser acupressure, transcutaneous electrical nerve stimulation (TENS) machines) that may be incorporated into their TMB sessions. In most cases, there was no distinction of these services from the provision of the TMB therapies because they could be smoothly integrated into the TMB services. Therefore, the consent issues described in this manuscript reflect these broader perspectives.

\section{METHODS}

\section{Sampling}

The interviews from which this material was drawn come from a larger combined-methods project about the training and practice of TMB therapies involving a population-level survey and in-depth interviews with practitioners practicing multiple TMB therapies, described in a separate manuscript ${ }^{(22)}$. Approval for the study was obtained from the University of Calgary Conjoint Health Research Ethics Board. In brief, from the 283 practitioners who volunteered for interviews, practitioners were purposively selected for maximum variation amongst the following variables: gender, municipality population type (categorized as urban, semi-urban, and rural), clinic focus or type (e.g., spa, private or group clinic), and types of TMB therapies practiced as reported by the practitioners ${ }^{(22)}$. Interviewed practitioners received a $\$ 40$ honorarium.

\section{Interview Process}

The lead author (AP) conducted all interviews, using a semistructured interview guide ${ }^{(19,22)}$. After obtaining informed consent, the interviews began with questions about the TMB training programs the practitioner had taken. Discussions then progressed to whether the practitioners combined their therapies during treatments, and if so, when and how that was done (an article specific to this in-depth exploration of the process of practice has been published $\left.{ }^{(19)}\right)$. This led to the question on the informed consent process: "If you are combining therapies like this, how do you negotiate consent?" This question assumed that the practitioners understood the ethical reasons that required them to engage in a consent process and, therefore, proceeded directly to how the practitioners engage into a consent process, not if or why. Probing questions helped explore the different consent 
processes the participants engaged in, such as initial consent and on-going consent.

\section{Analysis}

The interviews were taped, transcribed, and validated for accuracy of transcription. The computer program Atlas.ti was used for the analysis. Qualitative description was used to find, compare, organize, and link concepts and ideas within and across interviews because this method allows the researchers to stay close to a direct description of the data ${ }^{(23,24)}$. Ideas and concepts were identified in the recorded data, and codes using the participants' language were developed to categorize the ideas and concepts. The codes were iteratively refined and reduced to clarify and categorize the ideas and concepts. These codes were then clustered into themes, which were progressively modified and refined to best fit the data as the interviews continued. Common methods were used to develop and test the rigor of analysis ${ }^{(25-28)}$. Primary analysis was conducted by the lead author, and the coding process was reviewed by a second investigator. Interviews continued to be conducted until theoretical saturation, at which point no new insights or variations were arising from further interviews ${ }^{(29)}$.

\section{RESULTS}

\section{Participants}

The 19 participating practitioners had trained in up to $17 \mathrm{TMB}$ therapies (range 5-17; median 10), but typically used between two and ten therapies (median five) in their practices. Most had also taken introductory courses in other TMB therapies (i.e., initiatory classes that may include introduction to typical or beginner techniques), and also practiced non-TMB therapies. Descriptors of the participants are in Table 1.

All the practitioners described obtaining consent from their clients at the point of intake; verbal or written was not confirmed. In the interviews, discussions regarding consent usually began with descriptions of when and how informed consent arose. While exploring the reason why participants engage in obtaining informed consent was not a planned part of the interview questions, description of this issue often arose as context during the description of the consent process. Insight into why they engage in this process is covered in the section "Compelled to engage in informed consent" (below). The degree of engagement into fully informed consent is explained by the dichotomous themes described in the sections below, "I give them a general treatment goal" and "Would that be okay?"

\section{Compelled to engage in informed consent}

All practitioners, in their descriptions of the consent process with patients, revealed a fundamental understanding of informed consent. However, their concepts of informed consent did not translate into a single or concise reason for engaging in a consent process. At one extreme were the interviewees who engaged in a consent process primarily to comply with association membership or other mandatory routine requirements, such as those at places of employment. For example, Practitioner 17 said:

TABLE 1. Participant Characteristics $(n=19)$

\begin{tabular}{|c|c|}
\hline Characteristic & Detail \\
\hline Gender & Female $=15 ;$ Male $=4$ \\
\hline $\begin{array}{l}\text { Work setting } \\
\text { ( } \mathrm{n} \text {, not exclusive) }\end{array}$ & $\begin{array}{l}\text { Shared clinic }=4 \text {, private clinic }=6 \text {, home clinic }=4 \text {, salon }=1 \text {, fitness club }=1 \text {, spa }=4 \text {, chiropractic } \\
\text { clinic }=2 \text {, medical clinic }=1 \text {, outcalls } / \text { on-site }=1\end{array}$ \\
\hline Years in practice & Median: 10 ; range $3-30+$ years \\
\hline $\begin{array}{l}\text { Number of TMB therapies trained } \\
\text { in (not including introductions) }\end{array}$ & $\begin{array}{l}\text { Median: 10; range 5-17. } \\
\text { The TMB therapies practiced by the participants include: acupressure, Alexander Technique, aroma- } \\
\text { therapy, A.R.T., Aston patterning, Bowen, chair massage, Chi Nei Tsang, craniosacral therapy, Esalen, } \\
\text { gyrokinetics, hot/cold stones massage, hydrotherapy, Indian head massage, lomi lomi, manual lymph } \\
\text { drainage, massage therapy, maternal/pregnancy massage, myofascial release, Onsen, PNF, rebalanc- } \\
\text { ing, Raindrop Therapy }{ }^{\mathrm{TM}} \text {, reflexology, shiatsu, sports massage, St. John Neuromuscular Therapy, } \\
\text { structural integration, Swedish massage, Thai massage, trager, trigger point therapy, and Visceral } \\
\text { Manipulation }{ }^{\mathrm{TM}} \text {. }\end{array}$ \\
\hline $\begin{array}{l}\text { Number of introductory TMB } \\
\text { courses taken }\end{array}$ & Median: 2 ; range $0-5$ \\
\hline $\begin{array}{l}\text { Number who also practice } \\
\text { therapies that are not TMB (n) }\end{array}$ & $\begin{array}{l}\mathrm{n}=12 \\
\text { The non-TMB therapies described include: devices, bio-energy therapies (e.g., Reiki), nutrition, } \\
\text { hypnosis, ingested/topical products, systems approaches (shamanism, counseling). }\end{array}$ \\
\hline
\end{tabular}

\footnotetext{
"Introductory courses were not systematically pursued during the interviews. Some practitioners only described these using "a bunch",
} "some", "a few." These were taken to mean "more than one", and were quantified as 2 for the calculation of the median. 


\begin{abstract}
"The only reason I started to do the things that I do now is because I made a promise as a... person who belongs to [my professional association]. I have signed that I will adhere to a certain code of ethics and those require me to disclose and get permission, and tell the client what to expect. That's part of my commitment, I have to do that."
\end{abstract}

In contrast, other practitioners engage in a consent process from their commitment to the principles of patient autonomy. Practitioner 14, who uses an on-going consent process throughout treatment, described the practitioner-patient relationship and patient autonomy as a primary driver:

\begin{abstract}
"The tricky part about [on-going mutual consent] is that it can be a rationale for people doing all kinds of, you know, whatever they please. And there's also this question about how much can a person truly give informed consent when they are lying naked on a table. I think those are issues I'm always trying to pay attention to, particularly with the intense experiences I've had around working with people who have been abused at different levels and lose their ability to even advocate for their experience..."
\end{abstract}

Between these extremes, practitioners generally based their decision to engage in a consent process on the belief that it is a good thing for them, the patients, or both. How a practitioner engaged in consent, as described below, was not always directly linked with why they engaged in consent, though the practitioners who engaged in consent throughout the treatment session were more likely to have mentioned patient respect, autonomy, or shared decisions in their discourse relative to those who engaged in a general consent process. No consent process described by practitioners addressed explicitly confirming consent for every therapy applied in the session.

\section{"I give them a general treatment goal"}

"They're consenting to come in and allow me to manipulate their body. So, you know, whether I do it with Reiki, with massage, or with acupressure, I think it's not an issue." (Practitioner 2)

General consent, as introduced by the above quotation, is when practitioners do not specifically address consent for each provided therapy. Rather, the initial consent for treatment is understood to be sufficient for the session, and perhaps even for on-going treatment sessions. Practitioners described several underlying assumptions for such an approach: (a) the patient understands that the practitioner offers "bodywork" of some sort and that the patient does not need or want to know explicitly the individual TMB therapies or when different TMB therapies are being applied; (b) the patient knows that the practitioner provides a set of TMB therapies and expects the practitioner will choose whatever is most appropriate; or (c) the patient may not be distinguishing, or cannot distinguish, between the different types of TMB therapies being applied. It is not clear if these assumptions are ever addressed directly with the patients or if they represent any given patient's truth or preference regarding the delivery of care.

\section{"To my understanding and my belief these are all massage techniques. It all is kind of an umbrella of massage... So when they've come to me to be treated, they want me to do what needs to be done to make it better. They're trusting me to make that decision. So as far as informed consent, I don't ask them, 'Is it okay if I do myofascial on you?' because that means nothing to a client. ... So I don't generally do that." (Practitioner 16)}

\begin{abstract}
"I think in general, some clients don't want to know anything and they just want to feel the end result, while other clients will ask and want to know. So I don't generally tell them unless the client seems to be inquisitive... In my informed consent I do with my clients, I don't specify that out. I give them a general treatment goal..." (Practitioner 1)
\end{abstract}

This is not to imply that practitioners doing a general consent process do not also elicit client feedback and input generally during the treatment process, but some may not specifically solicit consent during the variation and mixing of therapies that can occur during treatment application.

\section{"Would that be okay?"}

In contrast, some practitioners will mention a therapy as they apply it.
"What I do is I usually have people come in for general therapeutic massage. At some point when they're lying there, you work on their scalp and then I just do a little bit of Indian head massage. I do gentle stretching, which is Thai massage, so people aren't aware that they're getting the full-meal deal kind of thing. But I do say to them, 'You know this is a Thai stretch,'or 'This is Indian head massage, 'or such, so they're aware of what I'm doing. " (Practitioner 8)

Practitioners using this approach are alerting the patient to a change in therapy, ensuring a basic level of understanding, but not necessarily soliciting consent. Knowing that a practitioner provides a variety of therapies, coupled with knowledge of the therapies themselves, may give the patient the option to request or refuse therapies at the beginning or at any point during a session, but their perceived level of 
autonomy may be limited in this scenario, particularly if the practitioner is already applying the therapy.

A more nuanced form of on-going negotiation involves explaining changes or choices to the patient as they arise, if a shift in treatment needs to take place. Practitioner 15 describes two such scenarios:

\section{"I will maybe explain to them, 'I really think we need to do some fascial work on your shoulders. Now, it's going to take a little bit longer because the way I do it, it's not a forced thing. It's slow, so I maybe have to do a three quarter hour instead of a half hour. Would that be okay?' That kind of thing. If I'm adjusting a time or [therapy], like with cranial sacral, if I really think they'd benefit from that I'll say, 'You know, I really think you should try this. I think it could help you. Would you be willing to try that?"'}

Other practitioners don't think of consent specifically in terms of therapies that they provide, but rather consider consent for each component or moment of on-going treatment within a session. Comments about this form of consent were some of the most detailed responses about consent with regard to the consideration of the meaning inherent in receiving informed consent for treatment.

"I find that formal consent as it's taught is not practiced at all... I think there's a really important part of that language which is informed consent and I tend to focus on the informed part as leading into consent. ... The more that I can educate people to understand what it is that I'm doing, to give them some sense of why, like how that's connected, then what I find is that people can move with me. If I listen to their tissue response and check, 'Okay, how does that work? Does that feel like you can go with it?' 'if I ever have any question about what I'm reading [sensing in their tissues], then we find our sense of consent, of mutual agreement, arising naturally out of that approach. (Practitioner 14)

"I'll explain that I do physical and energetic work and I'm trying to work as much with their body as I possibly can. So the more we work together we're going to be communicating. I'm going to be asking "how do you feel about this?"' (Practitioner 15)

\section{DISCUSSION}

On paper, the process of obtaining informed consent seems straightforward. When applied, its complexity, as illustrated by the study findings, arises from multiple sources including variation in patient needs and desires, variation in practitioner motivations, comfort and habits, and the interaction between the two ${ }^{(6,8,30,31)}$. Not all practitioners are clearly ascertaining what degree of consent patients desire. Patients, and practitioners, may not realize that the consent process is potentially more complicated when multiple therapies are used. Additionally, the impact of regulatory requirements for consent in the case of multiple therapies can confuse things further-when are the "additional therapies" part of the scope of the practice or outside of it, thus requiring formal notice of the patient? From a safety perspective, whether truly informed consent can occur is questionable because there are few studies on the safety of TMB therapies generally or when multiple TMB therapies are mixed.

The practitioners who use a generalized consent process may not be obtaining a fully informed consent, if only because they did not ascertain the client's preference for the degree of information or desire for participation in on-going treatment choices. From descriptions of practitioners preferring this approach, three underlying assumptions were found and presented in the section "I give them a general treatment goal". Acting on those assumptions seemingly requires two premises: 1) that the patient's goal(s) is more important than knowledge about the therapy being applied, and 2) that the added TMB therapies have equal or lesser degrees of risk of harm than the therapy that the patient believes or expects they are receiving. The first, that the overall goal is more important than knowing the therapies applied, fits with what many of the practitioners describe as occurring in practice. It will take additional research to verify whether patients concur, and if so, the implications for an informed consent process. Given the lack of documented harm from the execution of TMB therapies, the second assumption has yet to be challenged in any substantial way. However, without clear knowledge of the inherent risks of harm, and often only the practitioner's or profession's body of experiential knowledge to support harm or benefit, maintenance of patient autonomy is difficult and the possibility of a fully-informed consent is limited. Recommendations in this situation suggest an informed decision-making process is appropriate (discussions of pros and cons of the choices available, incorporating the patients beliefs and values) ${ }^{(32)}$. Therefore, use of an initial, generalized "blanket" consent will not adequately address informed consent or patient autonomy. Practitioners who obtain a general consent because they are required to do so are not necessarily meeting their professional obligations to the patient.

The process of practice is itself a complex phenomenon involving ongoing assessment and treatment choices, where practitioners may not even be consciously aware that they have mixed therapies or techniques, or may be using multiple simultaneous assessment inputs ${ }^{(19)}$. From this perspective, a blanket consent process would allow them to remain focused 
on addressing their immediate treatment situation. Additionally, a blanket consent seems practical, as a strictly applied consent process would require patients be informed of what is and is not known about each of the therapies applied by practitioners during the session, and would need the patient to choose which, when, and how each were applied. However, with a blanket consent, the ability to maintain patient autonomy is lost. If informed consent is going to be achieved, practitioners would need to recognize or define the points during the treatment process when it is appropriate or necessary to elicit consent. Dr. Paiva, a bioethicist and TMB educator, has addressed this issue to some extent. According to her, a morally valid health care choice by a patient "is not a form signed by the patient... A morally valid choice is a living, continuous dialogue: it involves an ongoing educational and relational process. The therapist continues to educate and dialogue with the patient throughout the therapeutic relationship to ensure that the patient has sufficient information and the opportunity to ask questions and find out what they need to know in order to ensure that the decision that he or she makes is his or her own personal choice. If the patient chooses to consent, confirmation of the continuing consent may be necessary or even required in recognition of the ongoing nature of the process and experience." (p. 190) ${ }^{(15)}$

Such a moment-by-moment approach is appropriate to the fluidity of the practice of multiple TMB therapies; consent in such a situation is not otherwise explicitly addressed in the TMB literature. Given the diversity of practitioners interviewed, the practitioners' statements suggest that understanding and applying consent knowledge to the extent described by Paiva has not been generally achieved within the TMB population from which these practitioners were chosen. For those practitioners who inform their patients when they choose to add in or change to additional therapies, this consent process would provide an opening for the patient to comment on or participate in the choice for the on-going application of the therapy. However, such consent is constrained because the treatment is ongoing and the practitioner is either recommending the therapy in the moment without a clear avenue for discussion or may already be applying it. Patients may not be concerned, however, because they are experiencing a continuity of bodywork, may not care about differentiating the types of bodywork they are receiving, or they may be focused on receiving therapy for a goal regardless of how it is achieved.

All the interviewed practitioners described at least some of the consent features in their process with patients, including on-going validation of the patients' experiences and needs. However, there was a distinct lack of agreement on what the process of consent may look like in the light of the practice of multiple therapies and how best to engage in that process. Given the potential similarity between some TMB techniques, and that most practitioners are trained in multiple therapies and may consciously and unconsciously mix therapies and treatments during sessions $^{(22)}$, clarification regarding the management of ongoing consent should be an important component of any TMB body of knowledge.

What is particularly striking about these results and conclusions is that the same issues were found in the research of ethics and consent in physiotherapy, a profession whose treatment delivery shares many similarities with TMB. While physiotherapists and their colleagues have been exploring consent issues since 1984, it is only more recently that issues in the practical application of consent in physiotherapy have been explored ${ }^{(30,31,33,34)}$. Specifically, research in physiotherapy indicates the theoretical basis of patient autonomy is well understood, along with the legal and professional imperatives for providing informed consent ${ }^{(2,34)}$. However, education regarding informed consent and subsequent regulatory demands tend to produce practitioners who look upon informed consent as rote duty or a process of conveying information, and the consent process primarily occurs early in the treatment process ${ }^{(30,31)}$. Relatively few practitioners ascertain a patient's knowledge or comprehension about all treatments suggested or provided, engage in shared decision-making, or use on-going consent confirmation ${ }^{(31)}$. Moreover, there is concern that an on-going consent process is difficult because it requires patients to make instantaneous informed decisions during treatment ${ }^{(31)}$. While it is acknowledged that the physiotherapists are meeting their legal duty to obtain informed consent, Fenety and colleagues' recommendation for physiotherapy is that consent should be conceived and acted on from an understanding-based perspective that entails using clear treatment goals and plans, on-going description of treatment process and choices, and verbal affirmation from patients ${ }^{(31)}$. Such an understanding-based consent process does not require full knowledge and understanding of all facets of the treatment, as patients will likely never have a level of expertise similar to the practitioner (i.e., an information differential with the practitioner maintaining greater knowledge), and does not require stopping treatment to develop full consent, which could be counterproductive ${ }^{(30)}$.

Understanding-based consent is an on-going process, and does require a higher degree of consent engagement at points that may be particularly relevant to patients, such as when their experience or the risk/benefit ratio of a treatment might change. This approach seems more realistic than explicit ongoing consent or shared decision-making ${ }^{(31,35,36)}$. Given that this TMB consent study found the same patterns of practice and consent as were described in the physiotherapy research, those physiotherapy recommendations also seem the most appropriate for practitioners of TMB therapies. 


\section{CONCLUSION}

While this research has attempted to critically explore gaps in these practitioners' consent processes, there are a few limitations to consider. These results are derived from a couple questions in a larger discourse with practitioners about their training and practice in the context of practitioners with training in multiple therapies. Some consent concepts that could have been explored, such as practitioners' training in shared decision-making and the implications of the power differential in the consent process- "how much can a person truly give informed consent when they are lying naked on a table (Practitioner 14)" were not. While these concepts surely underlie the themes developed and some of the approaches and thoughts expressed by the practitioners, they were not teased out as they could have been in a project devoted explicitly to the barriers and facilitators of informed consent processes. Additionally, the constraints of qualitative work must be remembered: the interviews are of 19 practitioners being interviewed on their training and practice. Their recall of this potentially unexpected topic - their consent processes - could be incomplete and self-censored, though the direct and honest statements seem to indicate a willingness to reflect on their own practice and respond truthfully.

How transferable are the results? The practitioners were chosen for maximum variation from among almost 300 volunteers in the province with different practices types, therapies, genders, and population environments; the themes were developed to encompass the diversity of responses. It is hoped, therefore, that the results reflect knowledge and truth about the population they were drawn from, but any comparison with other populations must be carefully considered. Finally, while the practitioners were given an opportunity to review the project results presented in the previous articles ${ }^{(19,22)}$, confirmation of these results was not obtained.

For most TMB therapies, the formal development of each therapy's professional body and the concomitant development of standards of practice are relatively new and the professional status for each is still evolving. If the results of this study seem relevant to other local populations or indicate limitations in any TMB professions' competency requirements, we would suggest some simple remedial steps. Educators of TMB therapies can address the challenge of teaching the implementation and the moral obligation of an informed consent process, not only from the perspective of the particular therapy they are teaching, but also from the broader perspective of a patient receiving $\mathrm{TMB}$ from a practitioner who will likely practice a variety of TMB therapies. Consent education should center on accomplishing on-going, understanding-based consent using the knowledge and experience of the practitioners, grounded in concern for the patient's autonomy, safety, and ethical well-being. For practitioners already practicing, professional organizations should develop, and make available, ethics programs and literature that address consent issues to this depth, and that specifically address the process of informed consent for multiple TMB-trained practitioners. This would include both how they describe and hold their practitioners to obtaining consent - moving from proof of consent to being accountable for a process of consent - and making safety profiling information on their therapies freely available to practitioners and patients. Given the lack of research regarding TMB consent processes, focused research about the process and effective forms of consent in TMB, such as the understanding-based consent suggested here, is much needed.

\section{ACKNOWLEDGMENTS}

This study could not have been done without the many participants, and especially the 19 practitioners who were willing to thoughtfully reflect on their training and practice experiences. Thank you. This project was funded by the Massage Therapy Foundation.

\section{CONFLICT OF INTEREST NOTIFICATION}

The authors are not aware of any conflicts of interest or potential conflicts of interest regarding the material in this manuscript. The Massage Therapy Foundation, funder of the study, had no role in the design of the study, in the collection, analysis and interpretation of the data, in the writing of the resultant articles, or in the decision to submit this article for publication. While the lead author is currently the Executive Editor of the IJTMB, the article was appraised by the Practice Section Editor and anonymously reviewed like any other IJTMB article.

\section{COPYRIGHT}

Published under the CreativeCommons AttributionNonCommercial-NoDerivs 3.0 License.

\section{REFERENCES}

1. Varricchio CGC, Jassak PFP. Informed consent: an overview. Semin Oncol Nurs. 1989;5(2):95-98.

2. Elkin S. Informed consent: requirements for legal and ethical practice. Physiother Theory Pract. 2001;17(2):97-105.

3. Mazur G. The historical development of the principle of free and informed consent. In: Mazur, G, editor. Informed Consent, Proxy Consent, and Catholic Bioethics. Food for the Good of the Subject. Informed Consent; 2012. p.1-12.

4. Mazur DJD. Informed consent: court viewpoints and medical decision making. Med Decis Making. 1986;6(4):224-230. 
5. Kirby MD. Informed consent: what does it mean? J Med Ethics. 1983;9(2):69-75.

6. Sim J. Informed consent and manual therapy. Man Ther. 1996;2(2):104-106.

7. Porcino A, Macdougall C, Mah R. A Recommendation for the Regulation of Massage Therapy Under the Health Professions Act, 1st ed. Edmonton, AB: Association of Massage Therapists and Wholistic Practitioners; 2006.

8. Williams JR. Medical Ethics Manual, 2nd ed. [Internet]. Axbridge, UK: World Health Communication Associates; 2009. Available from: http:// www.wma.net/en/30publications/30et hicsmanual/pdf/ethics manual en.pdf

9. NCBTMB. Standards of Practice [Internet]. Oakbrook Terrace, IL; 2009. [cited 11 Jan 2014]. Available from: http://www. ncbtmb.org/standards-practice

10. Fong B, Lavery ME, Pierce K, Salvo SG, Schenkman S, Schmidt DM, et al. Massage Therapy Body of Knowledge, 1st ed. Evanston, IL: MTBOK Task Force; 2010.

11. College of Massage Therapists of British Columbia. Bylaws of the College of Massage Therapists of British Columbia [Internet]. Vancouver, BC: CMTBC; 2013. Available from: http:/www.cmtbc.ca/sites/default/files/2013-11-01-CMTBCConsolidated-Bylaws.pdf

12. American Massage Therapy Association. Standards of Practice [Internet]. Evanston, IL: AMTA; 2014 [cited 11 Jan 2014]. Available from: http://www.amtamassage.org/About-AMTA/ standards.html

13. Massage Therapist Association of Alberta. Standards of Practice, 1st ed. [Internet]. Red Deer, AB: MTAA; 2011 [cited 2014 Jan 11]. Available from: http://www.mtaalberta.com/doc/ Governing_Documents/2012_Standards_of_Practice.pdf

14. Benjamin BE, Sohnen-Moe C. The Ethics of Touch. Philadelphia, PA: Lippincott Williams \& Wilkins; 2003.

15. Paiva C. Keeping the Professional Promise. Hamilton, ON: MT Pub.; 2004.

16. Yardley-Nohr T. Ethics for Massage Therapists. Philadelphia, PA: Lippincott Williams \& Wilkins; 2007.

17. McIntosh N. The Educated Heart: Professional Boundaries for Massage Therapists and Bodyworkers. Philadelphia, PA: Lippincott Williams \& Wilkins; 2010.

18. AMTA. Ethics [Internet]. Evanston, IL: American Massage Therapy Association; 2013 [cited 28 April 2013]. Available from: http://www.amtamassage.org/courses/listing. html?CategoryId $=2$

19. Porcino AJ, Boon HS, Page SA, Verhoef MJ. Exploring the nature of therapeutic massage bodywork practice. Int $J$ Ther Massage Bodywork. 2013;6(1):15-24.

20. Caspi O, Shalom T, Holexa J. Informed consent in complementary and alternative medicine. eCAM. 2011;2011:170793.
21. Caspi O, Holexa J. Lack of standards in informed consent in complementary and alternative medicine. Complement Ther Med. 2005;13(2):123-130.

22. Porcino AJ, Boon HS, Page SA, Verhoef MJ. Meaning and challenges in the practice of multiple therapeutic massage modalities: a combined methods study. BMC Complement Alt Med. 2011;11:75.

23. Sandelowski M. Whatever happened to qualitative description? Res Nurs Health. 2000;23(4):334-340.

24. Neergaard MA, Olesen F, Andersen RS, Sondergaard J. Qualitative description - the poor cousin of health research? BMC Med Res Meth. 2009;9:52.

25. Corbin JM, Strauss A. Grounded theory research: procedures, canons, and evaluative criteria. Qual Sociol. 1990;13(1):3-21.

26. Strauss A, Corbin JM. Basics of Qualitative Research: Grounded Theory Procedures and Techniques, 1st ed. Newbury Park, CA: Sage Publications, Inc; 1990.

27. Creswell JW. Research Design. Thousand Oaks, CA: Sage Publications, Inc; 2003.

28. Saldaña J. The Coding Manual for Qualitative Researchers, 1st ed. Thousand Oaks, CA: Sage Publications, Inc; 2009.

29. Sandelowski M. Sample size in qualitative research. Res Nurs Health. 1995;18(2):179-183.

30. Delany CM. In private practice, informed consent is interpreted as providing explanations rather than offering choices: a qualitative study. Aust J Physiother. 2007;53(3):171-177.

31. Fenety A, Harman K, Hoens A, Bassett R. Informed consent practices of physiotherapists in the treatment of low back pain. Man Ther. 2009;14(6):654-660.

32. Whitney SN, McGuire AL, McCullough LB. A Typology of shared decision making, informed consent, and simple consent. Ann Intern Med. 2004;140(1):54-59.

33. Purtilo RB. Applying the principles of informed consent to patient care. Legal and ethical considerations for physical therapy. Phys Ther. 1984;64(6):934-937.

34. Delany CM. Respecting patient autonomy and obtaining their informed consent: ethical theory-missing in action. Physiother. 2005;91(4):197-203.

35. Coulter AA. Partnerships with patients: the pros and cons of shared clinical decision-making. J Health Serv Res Policy. 1999;2(2):112-121.

36. Charles C, Gafni A, Whelan T. Shared decision-making in the medical encounter: What does it mean? (or it takes at least two to tango). Soc Sci Med. 1997;44(5):681-692.

Corresponding author: Antony J. Porcino, PhD, UBC School of Nursing, T201 - 2211 Wesbrook Mall, Vancouver, BC V6T 2B5, Canada

E-mail: antony.porcino@nursing.ubc.ca 